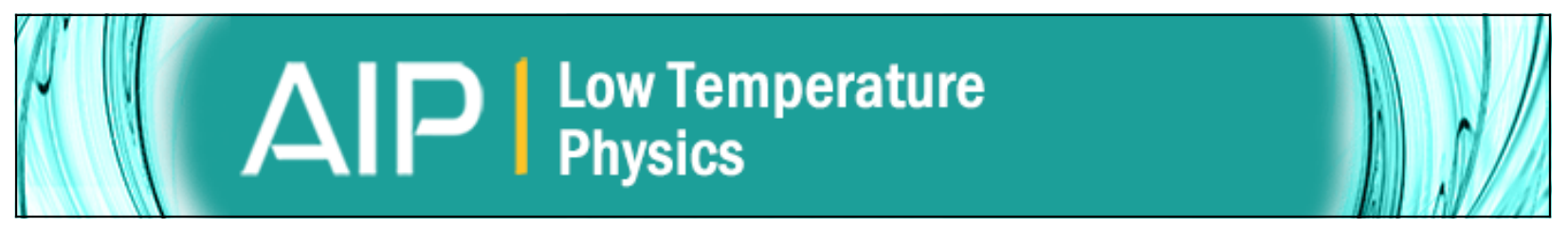

\title{
An effect of the curvature induced anisotropy on the spectrum of spin waves in a curved
} magnetic nanowire

V. S. Tkachenko, A. N. Kuchko, and V. V. Kruglyak

Citation: Low Temperature Physics 39, 163 (2013); doi: 10.1063/1.4792133

View online: http://dx.doi.org/10.1063/1.4792133

View Table of Contents: http://scitation.aip.org/content/aip/journal//tp/39/2?ver=pdfcov

Published by the AIP Publishing

Articles you may be interested in

Spin-torque effect on spin wave modes in magnetic nanowires

Appl. Phys. Lett. 101, 072409 (2012); 10.1063/1.4747152

Attenuation of propagating spin wave induced by layered nanostructures

Appl. Phys. Lett. 100, 132411 (2012); 10.1063/1.3699020

Anisotropic spin-wave patterns generated by spin-torque nano-oscillators

J. Appl. Phys. 109, 07 C733 (2011); 10.1063/1.3566000

Spin wave assisted current induced magnetic domain wall motion

Appl. Phys. Lett. 96, 242501 (2010); 10.1063/1.3446833

Spin waves in a periodically layered magnetic nanowire

J. Appl. Phys. 98, 014304 (2005); 10.1063/1.1935764

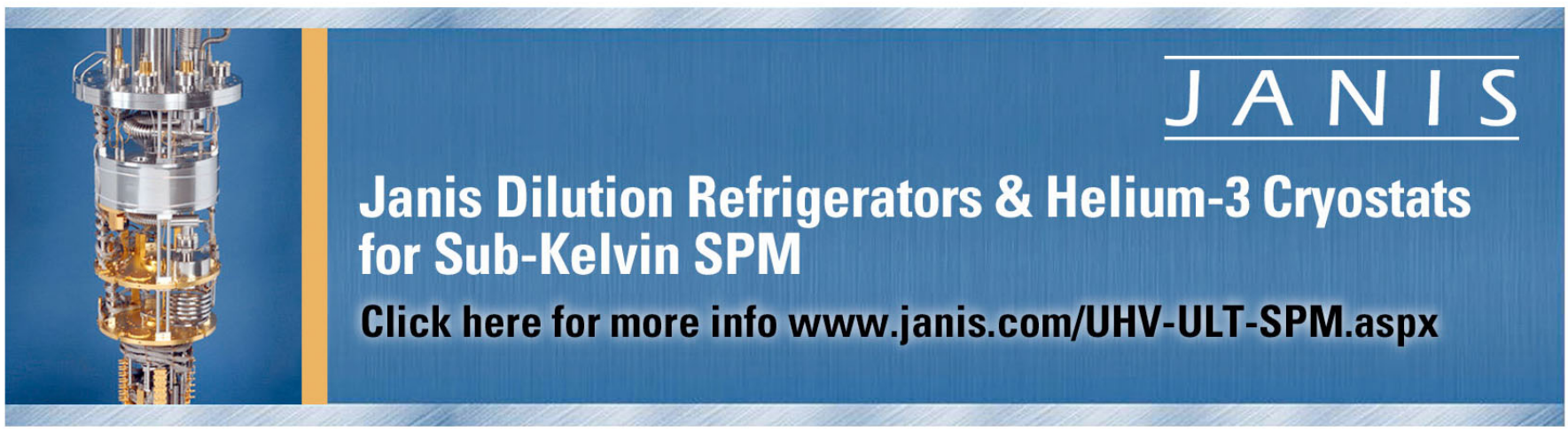




\title{
An effect of the curvature induced anisotropy on the spectrum of spin waves in a curved magnetic nanowire
}

\author{
V. S. Tkachenko a) and A. N. Kuchko \\ Donetsk National University, ul. Universitetskaya 24, Donetsk 83000, Ukraine \\ V. V. Kruglyak \\ School of Physics, University of Exeter, Stocker Road, Exeter EX4 4QL, United Kingdom
}

(Submitted June 22, 2012; revised August 28, 2012)

Fiz. Nizk. Temp. 39, 214-218 (February 2013)

Within the framework of the solid state theory, an expression for the spectrum of spin waves propagating in a thin magnetic nanowire curled into a helix (spiral) is obtained. Its modification under the effect of a periodic modulation of the helical pitch is analyzed. In particular, it is shown that the periodic modulation of the helix pitch leads to the appearance of band gaps in the spectrum of spin waves. The influence of the modulation depth of the helical pitch on a size of the first gap is considered. @ 2013 American Institute of Physics. [http://dx.doi.org/10.1063/1.4792133]

\section{Introduction}

An ability to create magnetic devices with an operational frequency close to the terahertz range has stimulated the development of the magnonics — an area of magnetism, which studies spin waves in general and in magnetic nanostructures in particular. ${ }^{1}$ The magnetic structures with a periodic modulation of spin-wave characteristics, the so-called "magnon crystals" have attracted particular attention of researchers, ${ }^{1-6}$ partly fueled by a wealth of knowledge gained in the field of research of various waves of a different nature in periodic structures.

In thin-film magnon crystals, i.e., magnetic multi-layers and superlattices, the processes of propagation of spin waves are well known for both ideal structures ${ }^{2-4}$ and structures containing various types of inhomogeneities and defects. ${ }^{7-11}$ Recently there is a large number of works devoted to the study of one- and two-dimensional planar magnon crystals formed by periodic structuring of monolayer magnetic films, most often yttrium iron garnet ${ }^{12,13}$ or transition metals. ${ }^{14-16}$

However, since in the magnon devices the topology is essential, it is necessary to study properties of connections between different parts of structural elements of magnonic devices and metamaterials. This gives rise to the renewed interest in the theoretical study of curved low-dimensional systems. ${ }^{17,18}$ Especially important is to consider losses caused by scattering on curved parts of magnon waveguides.

A similar situation occurs in curved low-dimensional quantum systems. In particular, in Refs. 19-21, a quantummechanical problem of motion of an electron along the curved surface was considered, and it was found that any deformation of the surface leads to an additional "geometric" potential.

In this paper, within the framework of a continuum medium approximation the propagation of spin waves is studied in curved nanowires which can act as magnon waveguides connecting structural elements of three-dimensional magneto-electronic devices for signal processing. ${ }^{22,23}$

\section{A model of the material}

Let us consider a curved magnetic nanowire bent in the form of a helix (spiral), as shown in Fig. 1.
A material of the wire can be described by the following parameters: the saturation magnetization $M_{0}$, the exchange constant $\alpha$, the uniaxial anisotropy $\beta$, and the gyromagnetic ratio $g$. We assume that an external field is absent, $H_{0}=0$, and the anisotropy axis is directed along the axis of the wire. A winding is characterized by the angle $\varphi$, helical pitch $h$; the radius of the cylinder, on the surface of which the spiral is located, is assumed to be constant and equal to $\rho$; the wire thickness is neglected.

\section{The spectrum of spin waves in a spiral magnetic nanowire}

Let us consider one of turns of the spiral. The dynamics of the magnetization is described by the Landau-Lifshitz equation,

$$
\frac{\partial \mathbf{M}}{\partial t}=-g\left[\mathbf{M} \times\left(\mathbf{h}_{m}+\beta(\mathbf{M} \boldsymbol{\mu}) \boldsymbol{\mu}+\alpha \Delta \mathbf{M}\right)\right]
$$

where $\boldsymbol{\mu}$ is a unit vector along the wire, the magnetostatic (demagnetizing) field $\mathbf{h}_{m}=-\hat{N} \mathbf{M}$, where $\hat{N}$ is the local tensor of demagnetizing coefficients, which can be introduced because of the small thickness of the wire.

Consider small deviations of magnetization from the ground state - the magnetization along the wire. For this, we represent the distribution of magnetization in the form, (a)

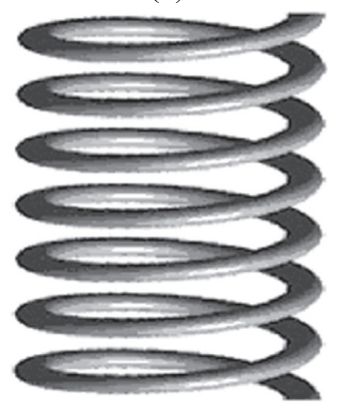

(b)

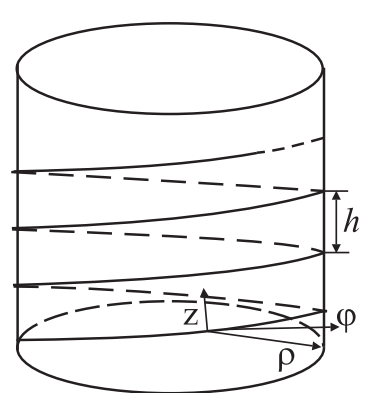

FIG. 1. A magnetic nanowire, curled into the shape of a helical (spiral) (a), and the coordinate system used in the calculations (b). 


$$
\mathbf{M}(\mathbf{r}, t)=\boldsymbol{\mu} M_{0}+\mathbf{m}(\mathbf{r}, t),|\mathbf{m}| \ll M_{0} .
$$

We linearize the Landau-Lifshitz equation (1), taking into account the relation $[\mathbf{M}(\mathbf{r}, t)]^{2}=M_{0}$, and by representing the variable part of the magnetization in the form of a Fourier component $\mathbf{m}(\mathbf{r}, t)=\mathbf{m}(\mathbf{r}) \exp \{i \omega t\}$, we obtain

$$
\begin{aligned}
i \omega \mathbf{m}= & -g\left[\boldsymbol{\mu} M_{0} \times(\alpha \Delta \mathbf{m}-\widehat{N} \mathbf{m})\right] \\
& -g\left[\mathbf{m} \times\left(\alpha M_{0} \Delta \boldsymbol{\mu}-\widehat{N} \boldsymbol{\mu} M_{0}+\beta M_{0} \boldsymbol{\mu}\right] .\right.
\end{aligned}
$$

Let us choose a coordinate system associated with the wire. For this we introduce the moving frame of the curve consisting of the tangent to the wire $\varphi$, the normal to the wire $\boldsymbol{\rho}$ and the binormal $\mathbf{z}=[\boldsymbol{\rho} \times \boldsymbol{\varphi}]$. In this coordinate system, the vector $\boldsymbol{\mu}$ is co-directional with $\boldsymbol{\varphi}$, so the term $\hat{N} \boldsymbol{\mu} M_{0}=-N_{z} M_{0} \mu_{z} \mathbf{z}-N_{\rho} M_{0} \mu_{\rho} \boldsymbol{\rho}=0$.

The Eq. (3) can be written as

$$
\begin{aligned}
i \omega \mathbf{m}= & -g\left[\boldsymbol{\varphi} M_{0} \times\left(\alpha \Delta \mathbf{m}+N_{z} m_{z} \mathbf{z}+N_{\rho} m_{\rho} \boldsymbol{\rho}\right)\right] \\
& -g\left[\mathbf{m} \times\left(\alpha M_{0} \Delta \boldsymbol{\varphi}+\beta M_{0} \boldsymbol{\varphi}\right] .\right.
\end{aligned}
$$

We set $\Omega=\omega /\left(g M_{0}\right)$. We represent the variable part of the magnetization in the form of an expansion in unit vectors $\mathbf{m}=m_{\rho} \boldsymbol{\rho}+m_{\varphi} \boldsymbol{\varphi}+m_{z} \mathbf{z}$ of the coordinate system. Using the representation of the Laplace operator in curvilinear coordinates, ${ }^{24}$ we obtain

$$
\begin{aligned}
\alpha \Delta \mathbf{m}= & \boldsymbol{\rho}\left(\Delta m_{\rho}+\delta \beta m_{\rho}+2 \delta \beta \frac{\partial m_{\varphi}}{\partial \boldsymbol{\varphi}}\right) \\
& +\boldsymbol{\varphi}\left(\Delta m_{\varphi}+\delta \beta m_{\varphi}-2 \delta \beta \frac{\partial m_{\rho}}{\partial \boldsymbol{\varphi}}\right)+\mathbf{z} \Delta \mathrm{m}_{z},
\end{aligned}
$$

where $\delta \beta=-\frac{\alpha}{\rho^{2}+(h / 2 \pi)^{2}}$, and it is denoted that $\Delta m_{\rho, \boldsymbol{\varphi}, z}=-\delta \beta \frac{\partial^{2} m_{\rho, \varphi, z}}{\partial \varphi^{2}}$.

Let us write down separately the value,

$$
\alpha \Delta \varphi=\delta \beta \varphi .
$$

We write the vector product,

$$
\alpha[\mathbf{m} \times \Delta \boldsymbol{\varphi}]=\left[\left(\boldsymbol{\rho} m_{\rho}+\phi m_{\varphi}+\mathbf{z m}_{z}\right) \times(\delta \beta . \varphi)\right],
$$

which can be reduced to the form

$$
\alpha[\mathbf{m} \times \Delta \varphi]=-\delta \beta\left(\rho m_{z}-\mathbf{z} m_{\rho}\right) .
$$

Thus, from the Eq. (4) one can obtain the following relation:

$$
\begin{aligned}
i \Omega \mathbf{m}= & N_{z} m_{z} \boldsymbol{\rho}-N_{\rho} m_{\rho} \mathbf{z}+\Delta m_{\rho} \mathbf{z}+\left(2 \delta \beta \frac{\partial m_{\varphi}}{\partial_{\varphi}}\right) \mathbf{z}-\Delta m_{z} \boldsymbol{\rho} \\
& +\delta \beta m_{z} \boldsymbol{\rho}+\beta m_{z} \boldsymbol{\rho}-\beta m_{\rho} \mathbf{z} .
\end{aligned}
$$

The separate equations for each component $\mathbf{m}$ can be written as:

$$
\begin{aligned}
& i \Omega m_{\rho}=N_{z} m_{z}-\Delta m_{z}+\delta \beta m_{z}+\beta m_{z}, \\
& i \Omega m_{\varphi}=0, \\
& i \Omega m_{z}=-N_{\rho} m_{\rho}+\Delta m_{\rho}-\beta m_{\rho} .
\end{aligned}
$$

Let us write the equations using the arc length $\ell$ as a coordinate. Because when the bend radius $\rho$ and the pitch $h$ of the considered nanowire are constant and independent of coordinates the values $\Delta m_{\rho, z}$ can be defined as

$$
\Delta m_{\rho, z}=-\delta \beta \frac{\partial^{2} m_{\rho, z}}{\partial \varphi^{2}}=\alpha \frac{\partial^{2} m_{\rho, z}}{\partial \ell^{2}},
$$

then the expressions for the components $\mathbf{m}$ take the form,

$$
\begin{aligned}
& i \Omega m_{\rho}=N_{z} m_{z}-\alpha \frac{\partial^{2} m_{\rho}}{\partial \ell^{2}}+\delta \beta m_{z}+\beta m_{z}, \\
& i \Omega m_{z}=-N_{\rho} m_{\rho}+\alpha \frac{\partial^{2} m_{\rho}}{\partial \ell^{2}}-\beta m_{\rho} .
\end{aligned}
$$

Let us define the spectrum of spin waves in the curved nanowire; for this we represent the magnetization in the form $m_{\rho, z}=m_{0 \rho, z} \exp (i k \ell)$. Substituting this dependence into the preceding relation, assuming $N_{z}=N_{\rho}=2 \pi$, we obtain the dispersion relation,

$$
\Omega^{2}=\left(2 \pi+\alpha k^{2}+\beta+\delta \beta\right) \times\left(2 \pi+\alpha k^{2}+\beta\right) .
$$

The quantity $\delta \beta$ can be considered as an additional "geometric" anisotropy induced by bending, which is similar to the results obtained in Ref. 21. In our problem the infinitely thin nanowire was considered, allowing to neglect inhomogeneities in the distribution of the magnetization in a section of the wire, and thus the quantity $\delta \beta$ obtained in the work depends only on the curvature of the bend and the wire pitch.

Fig. 2 shows the spectrum of spin waves (SWs), obtained by Eq. (8) for the curved nanowire for values typical for permalloy: the exchange parameter $\alpha=4.06 \times 10^{-12} \mathrm{~cm}^{2}$, the saturation magnetization $M_{0}=800 \mathrm{Oe}$, the exchange length $L_{\mathrm{ex}}=5.68 \times 10^{-7} \mathrm{~cm}$. The spectrum is plotted for $\beta=6$ and $\rho=5 L_{\text {ex }}$.

In Fig. 2 it is shown that with the increase of the helical pitch (as in the case of increasing the radius), the frequency

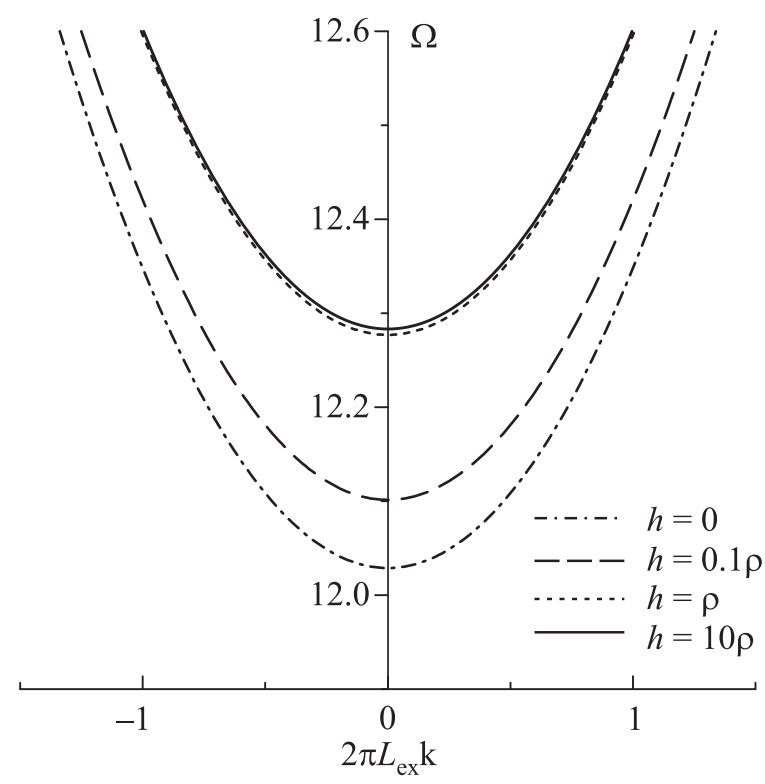

FIG. 2. A spectrum of spin waves in a homogeneous nanowire, curled into a spiral, for different values of the helical pitch $h$. 
of activation of SWs increases, asymptotically approaching the value characteristic of a direct nanowire. ${ }^{11}$

\section{A spiral magnetic nanowire with a periodic modulation of the pitch}

Since it is possible to create low-dimensional systems with a complex geometric shape, interesting is the problem of propagation of SWs in a magnetic nanowire, curled into the shape of a spiral with the modulated pitch.

Consider a wire, which can be represented in the form of two alternating parts. These parts can be characterized by the curvature radius $\rho_{1,2}$, the helical pitch $h_{1,2}$, the saturation magnetization $M_{0}$, the exchange constant $\alpha$, the anisotropy $\beta$ and the gyromagnetic ratio $g$; there is no external field $\left(H_{0}=0\right)$, the wire thickness is neglected. The length of each segment is assumed to be equal to $L_{1,2}$ $=n \sqrt{\left(2 \pi \rho_{1,2}\right)^{2}+h_{1,2}^{2}}, n$ is the number of turns of the wire.

In each segment of the curved nanowire the magnetization dynamics can be described using linearized LandauLifshitz equation,

$$
\frac{d^{2} m_{i}(z)}{d z^{2}}+k_{i}^{2}(z) m_{i}(z)=0, \quad i=1,2 .
$$

The wave vector $k_{i}$ can be found from the relation,

$$
\begin{aligned}
& \Omega^{2}=\left(2 \pi+\alpha k_{i}^{2}+\beta+\delta \beta_{i}\right) \times\left(2 \pi+\alpha k_{i}^{2}+\beta\right), \\
& \delta \beta_{i}=-\frac{\alpha}{\rho_{i}^{2}+\left(h_{i} / 2 \pi\right)^{2}},
\end{aligned}
$$

where $\Omega=\omega / g M_{0}, h=H / M_{0}, i=1,2$.

Using the method described in Ref. 25, one can obtain the expression for the spectrum of SWs,

$$
\begin{aligned}
\cos \left(k_{1} L_{1}\right) \cos \left(k_{2} L_{2}\right)+ & \left(\frac{k_{1}}{2 k_{2}}+\frac{k_{2}}{2 k_{1}}\right) \sin \left(k_{1} L_{1}\right) \sin \left(k_{2} L_{2}\right) \\
& =\cos \left(k\left(L_{1}+L_{2}\right)\right),
\end{aligned}
$$

where $k$ is the effective wave number, which is the reciprocal of the total phase shift in two different parts of the wire.

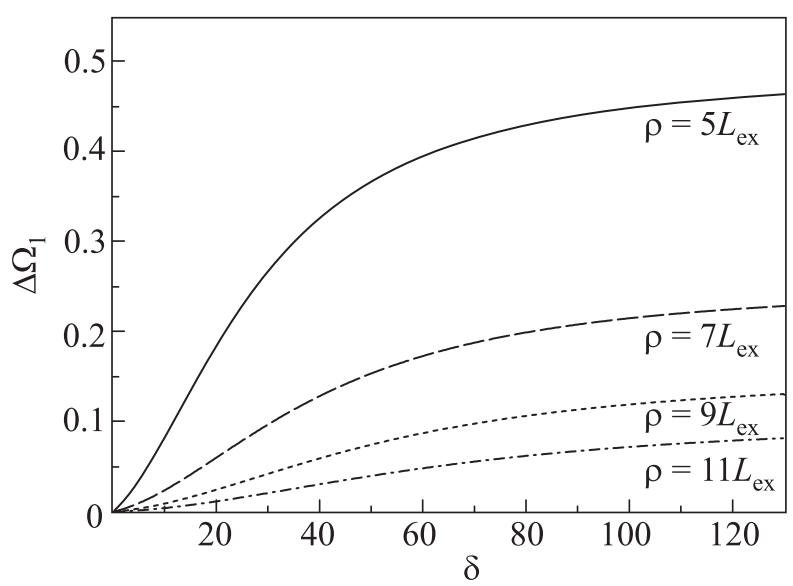

FIG. 3. A width of the first band gap as a function of the modulation depth of the helical pitch for different values of its radius $\rho$.
In this relation there are $L_{1}=n \sqrt{\left(2 \pi \rho_{1}\right)^{2}+h_{1}^{2}}$ and $L_{2}=n \sqrt{\left(2 \pi \rho_{2}\right)^{2}+h_{2}^{2}}$; the number of turns $n$ of the wire is the same on each of the segments.

This spectrum has a band structure. The magnitude of the band gaps can be determined from the condition $\left|\cos \left(k\left(L_{1}+L_{2}\right)\right)\right|>1$. Fig. 3 shows the size of the first gap $\Delta \Omega_{1}$ in the spectrum as a function of the ratio of change of the helical pitch to the exchange length $\delta=\left|h_{2}-h_{1}\right| / L_{\text {ex }}$. The dependence is plotted for $L_{\mathrm{ex}}=5.68 \times 10^{-7} \mathrm{~cm}, h_{1}=30 \mathrm{~nm}$, $\alpha=4.06 \times 10^{-12} \mathrm{~cm}^{2}, M_{0}=800$ Oe and $n=30$.

In Fig. 3, it can be seen that the pitch of the spiral affects the size of the band gaps. It is shown that the band gap increases with the pitch of the helix.

\section{Conclusions}

In this work, we derive an analytic expression for the spectrum of spin waves propagating in a thin magnetic nanowire, curled in the shape of a helix (spiral), and analyze its change under an effect of a periodic modulation of the helical pitch. In particular, we have shown that the periodic modulation of the pitch of the helix leads to the appearance of band gaps in the spectrum of spin waves. The influence of the modulation depth of the helical pitch on the size of the first gap is considered.

This work was supported in part by the project NoWaPhen (FP7 GA 247556).

a)Email: tkachenko_vera@i.ua

${ }^{1}$ V. V. Kruglyak, S. O. Demokritov, and D. Grundler, J. Phys. D: Appl. Phys. 43, 264001 (2010).

${ }^{2}$ S. A. Nikitov, P. Tailhades, and C. S. Tsai, J. Magn. Magn. Mater. 236, 320 (2001).

${ }^{3}$ M. Krawczyk, J.-C. Lévy, D. Mercier, and H. Puszkarski, Phys. Lett. A 282, 186 (2001).

${ }^{4}$ R. S. Iskhakov, S. V. Stolyar, M. V. Chizhik, and L. A. Chekanova, JETP Lett. 94, 301 (2011).

${ }^{5}$ B. Lenk, H. Ulrichs, F. Garbs, and M. Münzenberg, Phys. Rep. 507, 107 (2011).

${ }^{6}$ H. Al-Wahsh, A. Akjouj, B. Djafari-Rouhani, and L.G. Dobrzynski, Surf. Sci Rep. 66, 29 (2011).

${ }^{7}$ A. N. Kuchko, M. L. Sokolovskii, and V. V. Kruglyak, Physica B 370, 73 (2005).

${ }^{8}$ V. S. Tkachenko, V. V. Kruglyak, and A. N. Kuchko, J. Magn. Magn. Mater. 307, 48 (2006).

${ }^{9}$ V. A. Ignatchenko, Y. I. Mankov, and D. S. Tsikalov, J. Exp. Theor. Phys. 107, 603 (2008).

${ }^{10}$ Z. Danoyan, G. Piliposian, and D. Hasanyan, Waves Random Complex Media 19, 567 (2010).

${ }^{11}$ V. S. Tkachenko, V. V. Kruglyak, and A. N. Kuchko, Phys. Rev. B 81, 024425 (2010).

${ }^{12}$ S. L. Vysotskii, S. A. Nikitov, E. S. Pavlov, and Y. A. Filimonov, J. Commun. Technol. Electron. 55, 800 (2010).

${ }^{13}$ A. A. Serga, A. V. Chumak, and B. Hillebrands, J. Phys. D: Appl. Phys. 43, 264002 (2010)

${ }^{14}$ V. V. Kruglyak, P. S. Keatley, A. Neudert, R. J. Hicken, J. R. Childress, and J. A. Katine, Phys. Rev. Lett. 104, 027201 (2010).

${ }^{15}$ P. V. Bondarenko, A. Yu. Galkin, and B. A. Ivanov, Zh. Eksp. Teor. Fiz. 139, 1127 (2011)

${ }^{16}$ A. I. Marchenko and V. N. Krivoruchko, Fiz. Nizk. Temp. 38, 195 (2012) [Low Temp. Phys. 38, 157 (2012)].

${ }^{17}$ C. E. Zaspel and B. A. Ivanov, J. Magn. Magn. Mater. 286, 366 (2005).

${ }^{18}$ L. I. Magarill, D. A. Romanov, and A. V. Chaplick, Usp. Fiz. Nauk. 170, 325 (2000).

${ }^{19}$ R. C. T. da Costa, Phys. Rev. A 23, 1982 (1981).

${ }^{20}$ L. I. Magarill and M. V. Entin, Zh. Eksp. Teor. Fiz. 123, 867 (2003).

${ }^{21}$ L. I. Magarill, A. V. Chaplick, and M. V. Enetin, Usp. Fiz. Nauk 175, 995 (2005). 
${ }^{22}$ M. Dvornik, A. N. Kuchko, and V. V. Kruglyak, J. Appl. Phys. 109, 07D350 (2011).

${ }^{23}$ Y. Au, M. Dvornik, O. Dmytriiev, and V. V. Kruglyak, Appl. Phys. Lett. 100, 172408 (2012).

${ }^{24}$ S. P. Novikov and A. T. Fomenko, Elementy differentsialnoy geometrii $i$ topologii (Elements of Differential Geometry and Topology) (Nauka, Moscow, 1987).
${ }^{25}$ F. G. Bass, A. A. Bulgakov, and A.P. Tetervov, Vysokochastotnye svoystva poluprovodnikov so sverhreshetkami (High-Frequency Properties of Semiconductors with Superlattices) (Nauka, Moscow, 1989).

Translated by A. Sidorenko 